\title{
In Vitro and In Vivo Long-term Electrochemical Properties of Electrodes with Femtosecond-laser-induced Porosity for Visual Prostheses Based on Suprachoroidal Transretinal Stimulation
}

\author{
Hiroyuki Tashiro, ${ }^{1 *}$ Mariko Kuwabara, ${ }^{2}$ Yukari Nakano, ${ }^{2,3}$ Yasuo Terasawa, ${ }^{2,3}$ \\ Koji Osawa, ${ }^{4}$ Yurina Yoshimura, ${ }^{5}$ Haruna Doi,${ }^{6}$ and Jun Ohta ${ }^{2}$ \\ ${ }^{1}$ Department of Health Sciences, Faculty of Medical Sciences, Kyushu University, \\ 3-1-1 Maidashi, Higashi-ku, Fukuoka 812-8582, Japan \\ ${ }^{2}$ Graduate School of Materials Science, Nara Institute of Science and Technology, \\ 8916-5 Takayama-cho, Ikoma, Nara 630-0192, Japan \\ ${ }^{3}$ Artificial Vision Institute, Research and Development Division, Nidek Co., Ltd., \\ 13-2 Hama-cho, Gamagori, Aichi 443-0036, Japan \\ ${ }^{4}$ Artificial Retina Development Office, Development Division, Nidek Co., Ltd. \\ 34-14 Maehama Hiroishi-cho, Gamagori, Aichi 443-0038, Japan \\ ${ }^{5}$ Department of Health Sciences, Graduate School of Medical Sciences, Kyushu University, \\ 3-1-1 Maidashi, Higashi-ku, Fukuoka 812-8582, Japan \\ ${ }^{6}$ Department of Health Sciences, School of Medicine, Kyushu University, \\ 3-1-1 Maidashi, Higashi-ku, Fukuoka 812-8582, Japan
}

(Received August 8, 2017; accepted December 20, 2017)

Keywords: stimulation electrode, electrochemical impedance, charge injection capacity, retinal prosthesis, suprachoroidal transretinal stimulation

We developed a visual prosthesis based on suprachoroidal transretinal stimulation (STS) using electrodes with femtosecond-laser-induced porosity (FLiP electrodes). A current of 1.5 $\mathrm{mA}$ (1.25 times higher than that of a device under development) was applied by STS in six rabbits for 6 months to evaluate the long-term changes in the electrochemical properties of FLiP electrodes in vivo. The long-term stability of the FLiP electrodes was determined by in vitro and in vivo evaluations. The performance of the electrodes did not deteriorate after the long-term application of electrical stimulation in vivo. As no difference was observed between the in vivo electrochemical performance of the electrodes to which the stimulation current was and was not applied during the experiment, it is confirmed that the FLiP electrodes exhibit sufficient safety performance under long-term stimulation both in vivo and in clinical use. However, variations in the characteristics of the electrodes owing to the manufacturing method of the FLiP electrodes were observed. This variation should be reduced during the manufacturing process to avoid side effects owing to unexpected electrochemical behavior in clinical use. This result is useful in understanding the long-term safety testing results of STSbased retinal prostheses with FLiP electrodes.

*Corresponding author: e-mail: htashiro@med.kyushu-u.ac.jp http://dx.doi.org/10.18494/SAM.2018.1723 


\section{Introduction}

Recovery from blindness due to retinal disease is desired for many patients. Treatment for diseases such as age-related macular degeneration (AMD) and retinitis pigmentosa (RP) is still not available. Therapeutic approaches to replacing the function of the retina damaged by these diseases with artificial devices are being investigated and developed worldwide. ${ }^{(1)}$ The principle of these treatment methods is to obtain light sensation via electrical stimulation of the remaining visual nervous system. Some visual prostheses have already been successfully used in clinical applications. Retinal prostheses can be classified into epiretinal, ${ }^{(2)}$ subretinal, ${ }^{(3)}$ and suprachoroidal ${ }^{(4,5)}$ prostheses depending on the location of implantation. We developed retinal prostheses based on suprachoroidal transretinal stimulation (STS). ${ }^{(6)}$ Because the stimulation array is implanted into the scleral pocket, the STS approach is less invasive than other approaches. However, STS requires a larger charge injection to elicit light sensation than other approaches that use a method of direct contact with the retina. This is because, in STS, the stimulation electrode and the retina are relatively far from each other. ${ }^{(6-8)}$ We proposed a bullet-shaped electrode with a 3D surface as the stimulation electrode for STS. ${ }^{(9)}$ In addition, we developed high-performance electrodes with femtosecond-laser-induced porosity (FLiP electrodes). ${ }^{(10)}$ These electrodes achieved high performance by increasing the actual surface area relative to the $2 \mathrm{D}$ arrangement density and the geometric surface area. These FLiP electrodes are also used in devices aimed at commercial use. ${ }^{(1)}$ Although the long-term in vivo electrochemical behavior of stimulation electrodes with such a porous surface is complex and unknown, understanding the in vivo electrochemical characteristics of stimulation electrodes is important for ensuring safety and durability prior to clinical use. ${ }^{(12)}$ The purpose of this study is to obtain sufficient knowledge to evaluate the safety of STS-based visual prostheses in animal tests by clarifying the long-term in vivo electrochemical behavior of the FLiP electrodes.

\section{Materials and Methods}

Six Japanese white rabbits (2.5-3.5 kg, male, OrientalBioService, Kyoto, Japan) were used in this study (identified as Nos. 1-6). This animal study was approved by the Institutional Animal Care and Use Committee of Nidek Co., Ltd. All in vivo experiments were conducted in accordance with the Association for Research in Vision and Ophthalmology (ARVO) Statement for the Use of Animals in Ophthalmic and Visual Research. ${ }^{(13)}$ Statistical software (JMP Pro 13, SAS, USA) was used for the analysis.

\subsection{Stimulation electrode array}

An electrode array with two bullet-shaped platinum (Pt) electrodes on a $30-\mu \mathrm{m}$-thick Parylene substrate curved to fit the curvature of an eyeball was developed for this study (Fig. 1). The Pt electrodes exhibited a porous surface created by a femtosecond laser; therefore, we named them FLiP electrodes [Fig. 1(c)]. ${ }^{(10)}$ The electrodes were fabricated from a 1-mm- 


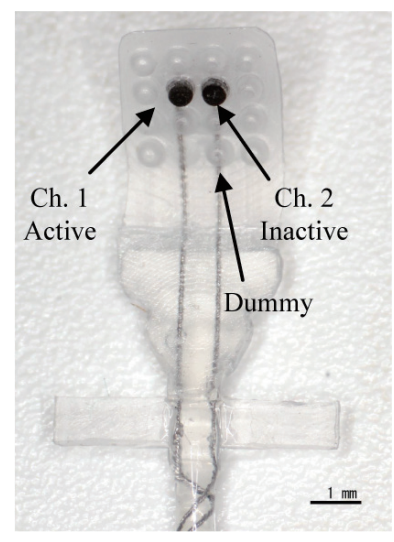

(a)

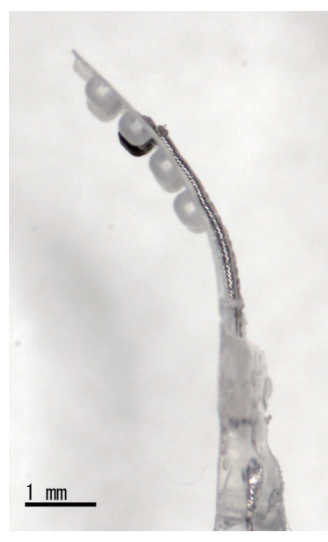

(b)

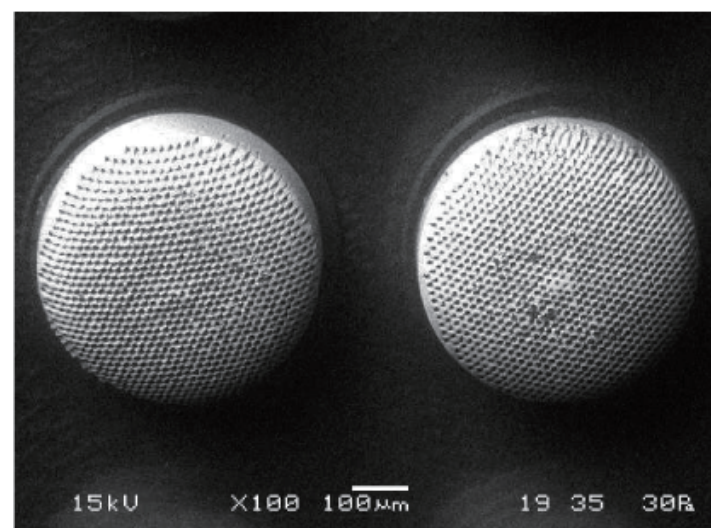

(c)

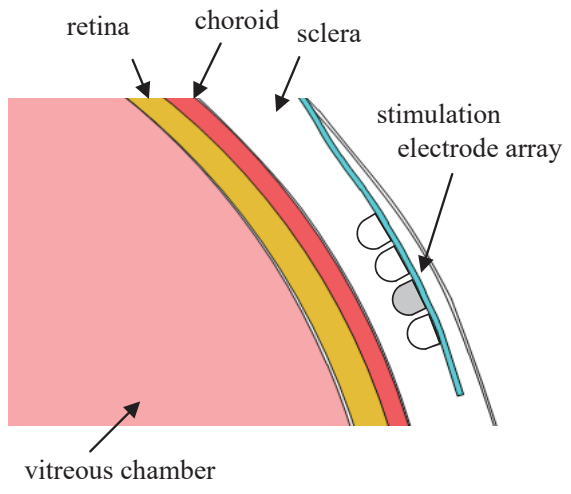

(d)

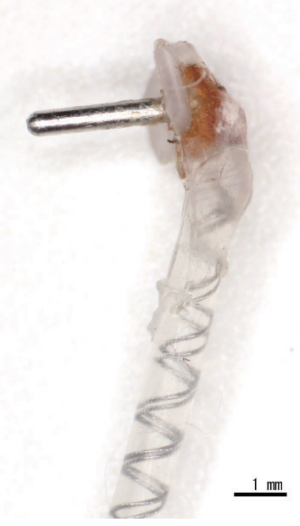

(e)

Fig. 1. (Color online) Electrode array with two FLiP electrodes. (a) Front view. (b) Side view. (c) Scanning electron microscope image of FLiP electrode. (d) Installation of stimulation electrode array based on STS. (e) Vitreous electrode as a return electrode.

diameter (dia.) Pt bar using a microlathe machine. ${ }^{(9)}$ The dia. and height of each electrode were 500 and $300 \mu \mathrm{m}$, respectively. The geometric surface area of the electrode was $0.00429 \mathrm{~cm}^{2}$. Femtosecond laser pulses were applied to the surface of a bullet-shaped Pt electrode to produce a porous surface. $^{(10)}$ The typical dia. and depth of the pore are 10 and $100 \mu \mathrm{m}$, respectively. ${ }^{(14)}$ Electrical stimulation was applied to one of the two electrodes, denoted as the "active" electrode [channel (ch.) 1 in Fig. 1(a)]. The other electrode was denoted as the "inactive" electrode; ch. 2 was not subjected to electrical stimulfation. The dummy electrodes fabricated from Parylene surrounded the two FLiP electrodes to reduce the mechanical stress between the electrode and the retina and choroid [Fig. 1(b)]. The vitreous electrode, as the return electrode, was fabricated from a Pt bar with a $3 \mathrm{~mm}$ length and $500 \mu \mathrm{m}$ dia. [Fig. 1(e)]. Prior to use, the stimulation electrodes were ultrasonically cleaned in ethanol for $5 \mathrm{~min}$. 


\subsection{Implantation of stimulation electrode array}

The electrode array was implanted into the sclera of the rabbits $(N=6)$. The implantation surgery was performed under 2.4\% isoflurane (Escain, Mylan, Tokyo, Japan) anesthesia. The inferior rectus and inferior oblique muscles were cut after conjunctival incision to expose the inferior surface of the sclera. A scleral pocket was then created precisely over the visual streak in the posterior pole area $9 \mathrm{~mm}$ from the corneal limbus. The stimulation electrode array was inserted into the flap [Fig. 1(d)], and the lead was sutured for fixation. The vitreous electrode [Fig. 1(e)] was inserted in the vitreous body via the pars plana.

\subsection{Electrical stimulation}

After 2 weeks of recovery, constant-current (CC) stimulation with a charge-balanced cathodic-first (CF) biphasic pulse $(1.5 \mathrm{~mA}$ amplitude, $500 \mu$ s duration, and $50 \mathrm{~Hz}$ repetition frequency) was applied to the active electrode for $8 \mathrm{~h}$ /day over 24 weeks. The test was conducted with the pulse duration used in the device under development. For the repetition frequency, the maximum value that can be set for the device was used. In consideration of the safety factor, the current value was tested at approximately 1.2 times the maximum current value that the device can output $(1.2 \mathrm{~mA})$. We used a long-term evaluation system with automatic stimulation and automatic measurement of the electrochemical impedance using freely moving rabbits. More details of the evaluation system are available in Ref. 15.

\subsection{Method of electrode cleaning after explantation}

Three of the explanted electrodes (Nos. 1-3) were cleaned by immersing them in $50 \mathrm{wt} \%$ potassium hydroxide (KOH; 000-63665, Kishida Chemical, Osaka, Japan) at $60{ }^{\circ} \mathrm{C}$ for $24 \mathrm{~h}$. The other three electrodes (Nos. 4-6) were cleaned by immersing them in an enzyme (Enz.) solution $\left(0.1 \mathrm{~g} / \mathrm{mL}\right.$ Bioplase AL-15-FG, Nagase ChemteX, Osaka, Japan) at $50{ }^{\circ} \mathrm{C}$ for $3 \mathrm{~h}$. We investigated whether the cleaning method affected the in vitro electrochemical properties after explantation. Measurement for 5 months after explantation was performed to determine whether any changes immediately after explantation were reversed spontaneously.

\subsection{Charge injection capacity (CIC)}

Before the implantation and after the explantation, the in vitro CICs of the FLiP electrodes were measured in $0.01 \mathrm{~mol} / \mathrm{L}$ phosphate-buffered saline [(PBS) (-); 164-18541, Wako Pure Chemical Industries, Osaka, Japan] under one atmosphere at room temperature (approximately $24{ }^{\circ} \mathrm{C}$ ) [Fig. 2(a)]. The CIC is the amount of charge that can be reversibly injected during a stimulation pulse with the electrode potential remaining within the water window $[-0.6$ to $+0.8 \mathrm{~V}$ vs silver/silver chloride $(\mathrm{Ag} / \mathrm{AgCl})$ reference electrode $(\mathrm{RE})$ for Pt electrode at $\mathrm{pH}$ 7.4] [Fig. 2(c)]. ${ }^{(12)}$ We designed and used a current source that can precisely control the charge balance and a unity gain amplifier that realizes high input impedance for the measurement. 


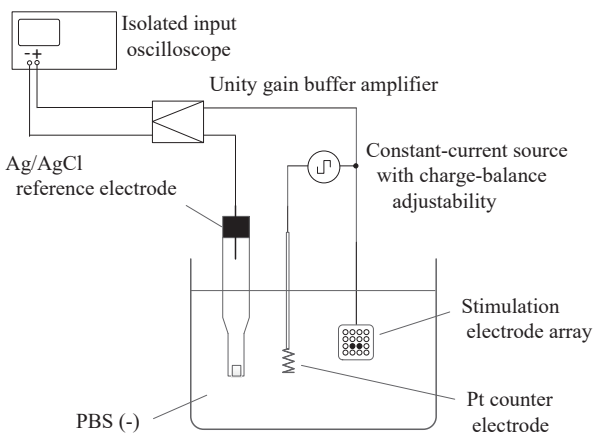

(a)

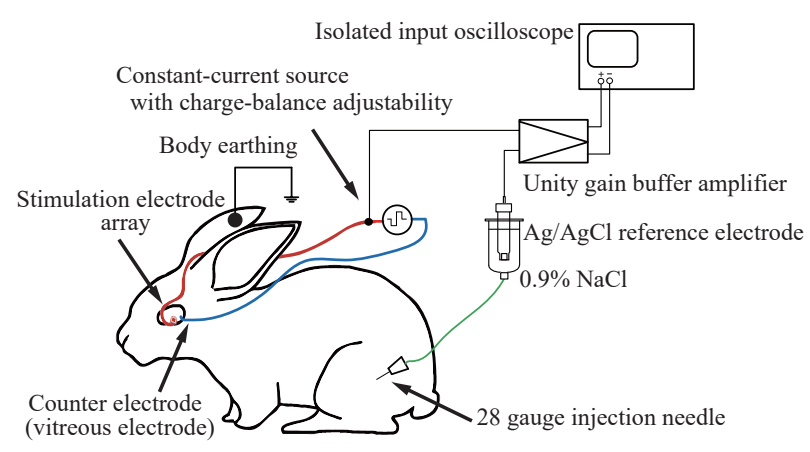

(b)

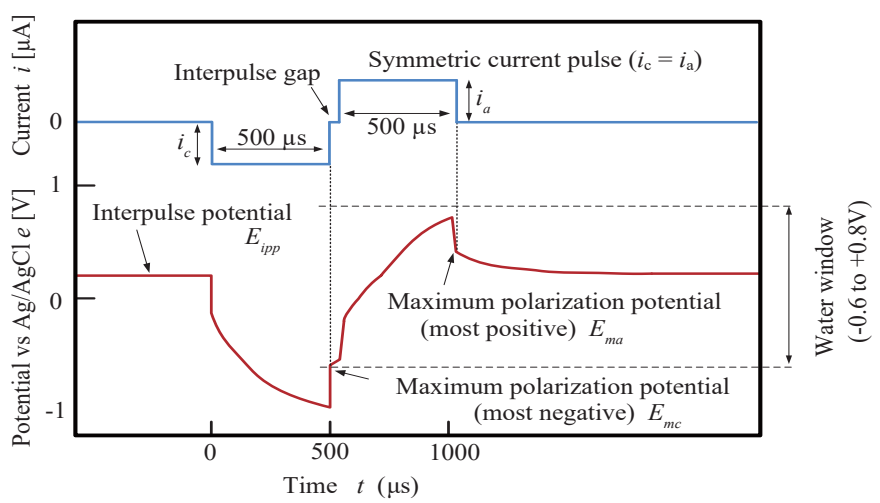

(c)

Fig. 2. (Color online) Experimental setup used in the measurement of CIC. (a) In vitro setup. The in vitro CIC was measured in $0.01 \mathrm{~mol} / \mathrm{L}$ PBS (-) under one atmosphere at room temperature. (b) In vivo setup. (c) Electrode potential across the Pt stimulation electrode in response to a biphasic symmetric current pulse with interpulse gap. The CIC is defined as the maximum injectable charge where the maximum polarization potential does not exceed the water window (typical value: -0.6 to $+0.8 \mathrm{~V}$ vs $\mathrm{Ag} / \mathrm{AgCl} \mathrm{RE}$ ).

The CF biphasic CC pulse with $500 \mu$ s duration and $30 \mathrm{~Hz}$ repetition frequency was applied between the stimulation electrode and the Pt-wire counter electrode (CE). The potential of the stimulation electrode with respect to the RE was observed through a unity gain buffer amplifier using an isolated input oscilloscope (DL750, Yokogawa Meters \& Instruments, Tokyo, Japan). Between the first cathodal pulse and the second anodal pulse, a period of $50 \mu$ s for opening the circuit was inserted as an interpulse gap. The same open-circuit period was provided even after the second pulse. The electrode potential immediately after stopping the current is the true polarization potential (PP), which does not include an ohmic drop. The maximum allowable current $I_{\max }$ for the maximum PP (MPP) on the cathodal side, $E_{m c}$, and the MPP on the anodal side, $E_{m a}$, without exceeding the water window was measured. The CIC was calculated by dividing the injected charge by the geometric surface area. Interpulse potential is the electrode potential before onset of the current pulse, which is governed by the oxidation state of the electrode surface. For the in vivo CIC measurements, the vitreous electrode was used as a $\mathrm{CE}$, and the $\mathrm{Ag} / \mathrm{AgCl} \mathrm{RE}$ was immersed in a salt bridge fabricated using a tube filled 
with physiological saline solution to establish an electrochemical connection with the rabbits [Fig. 2(b)]. The in vivo CIC was measured every month. For in vitro and in vivo CICs, a twoway repeated measures analysis of variance (rANOVA) was conducted to determine whether the CIC value depends on the active electrode, inactive electrode, or stimulation period. For the electrode cleaned with $\mathrm{KOH}$, a one-way rANOVA was conducted to determine whether the in vitro CIC and interpulse potential, $E_{i p p, c}$, before implantation, immediately after explantation, and after 5 months of explantation were different. After that, a paired $t$-test with Bonferroni correction was conducted to determine the groups that had significance. For the electrode cleaned with the Enz. solution, a paired $t$-test was conducted to determine whether the in vitro CIC and interpulse potential, $E_{i p p, c}$, before implantation, and immediately after explantation were different.

\subsection{PP}

PPs in vitro and in vivo were measured when a stimulus current of $1.2 \mathrm{~mA}$ was applied. The measurement setup is the same as that of the CIC measurement (Fig. 2). Regarding in vitro and in vivo PPs, a two-way rANOVA was conducted to determine whether the value depends on the active electrode, inactive electrode, or stimulation period. For the electrode cleaned with $\mathrm{KOH}$, a one-way rANOVA was conducted to determine whether the in vitro $E_{i p p, m}, E_{m c}$, and $E_{m a}$ with the application of a $1.2 \mathrm{~mA}$ current pulse before implantation, immediately after explantation, and after 5 months of explantation were different. For the electrode cleaned with the Enz. solution, a paired $t$-test was conducted to determine whether the in vitro $E_{i p p, m}, E_{m c}$, and $E_{m a}$ before implantation and after explantation were different.

\subsection{Electrochemical impedance}

The automatic measurements of the in vivo electrochemical impedance spectrogram (EIS) of each electrode with a two-electrode cell setup were performed while stimulation was suspended using a potentiostat/galvanostat equipped with a frequency response analyzer (FRA; AUTOLAB PGSTAT32, Metrohm, Utrecht, Netherlands). The CE, fabricated using a $0.5-\mathrm{mm}$-dia. Pt wire with a surface area greater than 1 $\mathrm{cm}^{2}$, for the EIS measurements was subcutaneously implanted in the temporal region on the opposite side of the stimulated eye. The measurements of the in vitro EIS were performed with a three-electrode cell setup in $0.01 \mathrm{~mol} / \mathrm{L}$ PBS (-) under one atmosphere at room temperature (approximately $24{ }^{\circ} \mathrm{C}$ ). The measurement system for the EIS is the same as the CIC measurement system shown in Fig. 2(a). A Ag/ $\mathrm{AgCl}$ electrode was used as an RE. The EIS measurement conditions were as follows: $10 \mathrm{~Hz}-10 \mathrm{kHz}, 20$ $\mathrm{mV}_{\text {rms }}$ constant-voltage (CV) sine wave. From the results of the EIS, statistical analysis was performed on the weekly and monthly values measured at $10 \mathrm{~Hz}, 100 \mathrm{~Hz}, 1 \mathrm{kHz}$, and $10 \mathrm{kHz}$. The weekly values were obtained by averaging the measured values for each day, excluding inaccurate data. For the impedance values at each frequency, a twoway rANOVA was conducted to determine whether the value depends on the active electrode, 
inactive electrode, or stimulation period. Additionally, a one-way rANOVA was conducted to determine whether the value of each impedance depends on the stimulation period. Moreover, correlation analysis was performed on the relationship between each impedance value and the stimulation period.

\section{Results}

Table 1 shows a summary of the results of the in vitro electrochemical properties before the start of in vivo experiments. The CIC of the FLiP electrode was approximately $1200 \mu \mathrm{C} / \mathrm{cm}^{2}$ in PBS (-). $E_{i p p}$ at the time of the CIC measurement was approximately $0.32 \mathrm{~V}$. When $E_{i p p}$ exceeded $0.4 \mathrm{~V}$, the non-Faradaic reaction was restricted on the anodal side [Fig. 3(a)]. There was no statistical difference in the in vitro performances between the active electrode and the inactive electrode before the start of in vivo experiments. From the beginning of the in vivo experiments, there were statistically significant differences in $E_{i p p, m}$ and $E_{m a}$ between electrode arrays Nos. 1-3 cleaned with $\mathrm{KOH}$ after explantation and Nos. 4-6 cleaned with Enz. solution after explantation.

The two-way rANOVA was conducted to confirm the changes in the electrochemical characteristics of the electrode cleaned with $\mathrm{KOH}$ before and after in vivo stimulation. There was no primary effect between the adaptation and nonadaptation of stimulation in vivo, although a primary effect was observed by comparing the results before implantation, immediately after explantation, and 5 months after explantation. For the changes before and after adaptation of the stimulus, no interaction with the adaptation/nonadaptation of stimulation was observed. As a primary effect and interaction with the adaptation/nonadaptation of stimulation were

Table 1

Summary of in vitro electrochemical properties of electrodes before the start of in vivo experiments. There are 12 electrodes in all $(N=12)$. These analyses were conducted to confirm that there were no differences in the values between the comparison groups before the start of in vivo experiments. All values in the table are in vitro values before the start of in vivo experiments. In the in vivo test, the electrode with application of the stimulation is denoted "active" $(N=6)$, and the electrode without application of the stimulation is denoted "inactive" $(N=6)$. Electrodes cleaned with $\mathrm{KOH}$ after in vivo testing were Nos. 1-3 ("KOH", $N=6$ ), and electrodes cleaned with Enz. solution were Nos. 4-6 ("Enz.", $N=6$ ). $C I C$ is the charge injection capacity. $E_{i p p, c}$ and $E_{i p p, m}$ represent interpulse potential at the time of CIC measurement and when applying $1.2 \mathrm{~mA}$ current, respectively. $E_{m c}$ is the MPP on the cathodal side, and $E_{m a}$ is the MPP on the anodal side, when the $1.2 \mathrm{~mA}$ current was applied. Independent $t$-tests were conducted to determine whether the numerical value differs between the "active" and "inactive" groups, and between cleaning methods (significant level $p=0.05$ ) before the start of in vivo experiments.

\begin{tabular}{lccccc}
\hline & $C I C\left[\mu \mathrm{C} / \mathrm{cm}^{2}\right]$ & $E_{i p p, c}[\mathrm{~V}]$ & $E_{i p p, m}[\mathrm{~V}]$ & $E_{m c}[\mathrm{~V}]$ & $E_{m a}[\mathrm{~V}]$ \\
\hline All & $1160 \pm 217$ & $0.320 \pm 0.117$ & $0.329 \pm 0.042$ & $0.173 \pm 0.049$ & $0.396 \pm 0.049$ \\
\hline Active & $\left.1130 \pm 175^{\mathrm{NS}}\right]$ & $0.348 \pm 0.092^{\mathrm{NS}}$ & $0.325 \pm 0.052^{\mathrm{NS}}$ & $0.176 \pm 0.064^{\mathrm{NS}}$ & $0.388 \pm 0.049^{\mathrm{NS}}$ \\
Inactive & $\left.1190 \pm 267^{\mathrm{NS}}\right]$ & $\left.0.293 \pm 0.141^{\mathrm{NS}}\right]$ & $\left.0.333 \pm 0.014^{\mathrm{NS}}\right]$ & $\left.0.168 \pm 0.034^{\mathrm{NS}}\right]$ & $\left.0.404 \pm 0.035^{\mathrm{NS}}\right]$ \\
\hline KOH, Nos. 1-3 & $\left.1200 \pm 230^{\mathrm{NS}}\right]$ & $0.351 \pm 0.156^{\mathrm{NS}}$ & $0.360 \pm 0.016^{*}$ & $0.196 \pm 0.043^{\mathrm{NS}}$ & $0.429 \pm 0.020^{*}$ \\
Enz., Nos. 4-6 & $\left.1120 \pm 216^{\mathrm{NS}}\right]$ & $\left.0.290 \pm 0.053^{\mathrm{NS}}\right]$ & $\left.0.298 \pm 0.036^{*}\right]$ & $\left.0.151 \pm 0.047^{\mathrm{NS}}\right]$ & $0.363 \pm 0.029^{*}$
\end{tabular}

Values are mean $\pm \mathrm{SD}$.

${ }^{*}=$ significant $(p \leq 0.05), \mathrm{NS}=$ not significant $(p>0.05$, independent $t$-test $)$. 


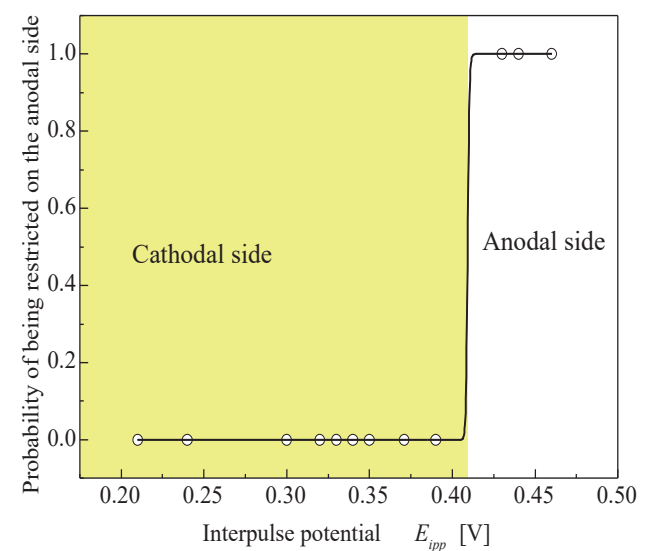

(a)

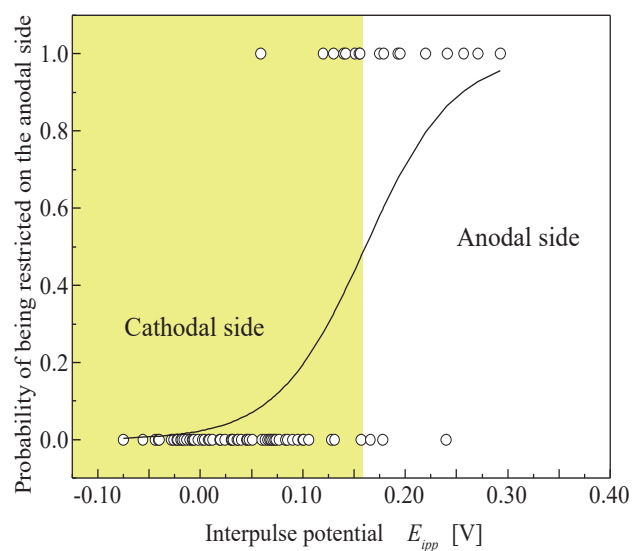

(b)

Fig. 3. (Color online) Result of logistic regression analysis of interpulse potential, $E_{i p p}$, whether the non-Faradaic reaction was restricted on the anodal side or the cathodal side during CIC measurement. The plot shows $E_{i p p}$ when the non-Faradaic reaction was restricted on the anodal side or the cathodal side at the time of actual CIC measurement [Fig. 2(c)]. The points are plotted as the probability of being restricted on the anodal side in the actual measurement; the probability is equal to 1 when restricted on the anodal side and equals 0 when restricted on the cathodal side. The lines represent the results of sigmoid curve fitting. (a) In vitro. (b) In vivo. When $E_{i p p}$ exceeded 0.4 $\mathrm{V}$ in vitro and $0.16 \mathrm{~V}$ in vivo, the non-Faradaic reaction was restricted on the anodal side.

not observed, the active and inactive electrodes were grouped together and compared before and after in vivo stimulation by one-way rANOVA. Figure 4 shows in vitro electrochemical evaluation values of the electrodes cleaned with $\mathrm{KOH}$ after in vivo stimulation. The $E_{i p p}$ value decreased after explantation; accordingly, CIC and $E_{m c}$ decreased as well. However, $E_{m a}$ increased after explantation. Although $E_{i p p}$ and $E_{m c}$ continued to decline, the changes in CIC and $E_{m a}$ recovered over time. As these changes were considered to be a result of $\mathrm{KOH}$ cleaning on the basis of the comparison with the results of the electrode cleaned with Enz. solution described later, a detailed analysis on impedance was not conducted.

The two-way rANOVA was conducted to confirm the changes in the electrochemical characteristics of the electrode cleaned with Enz. solution before and after in vivo stimulation. No primary effect was observed between the adaptation and nonadaptation of stimulation in vivo, except for $E_{m a}$. As a primary effect and interaction with the adaptation and nonadaptation of stimulation were not observed, except for $E_{m a}$, active and inactive electrodes were grouped together and compared before and after in vivo stimulation by one-way rANOVA. Because we focused on the temporal change, the analysis was conducted using repeated measures. The active and inactive electrodes are independent, even though they are on the same electrode array. Comparison of $E_{m a}$ on the active and inactive electrodes using a paired $t$-test gave a significant difference $(t=3.02, p=0.029)$, although there was no significant difference according to an independent $t$-test $(t=0.690, p=0.506)$. Before implantation, $E_{m a}$ of the inactive electrode is significantly higher than that of the active electrode $(t=7.26, p=0.018$, paired $t$-test). The difference between the inactive and active electrodes detected by two-way rANOVA is thought to be due to the difference existing before experimental use [Fig. 5(f)]. In fact, with respect to $E_{m a}$, no interaction was detected before/after and adaptation/nonadaptation 


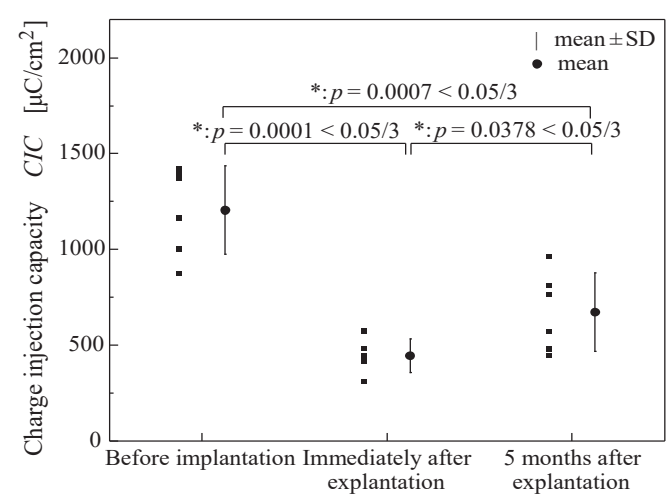

(a)

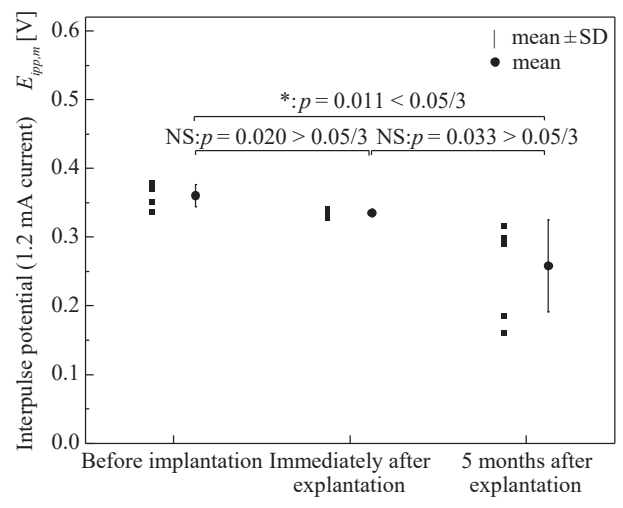

(c)

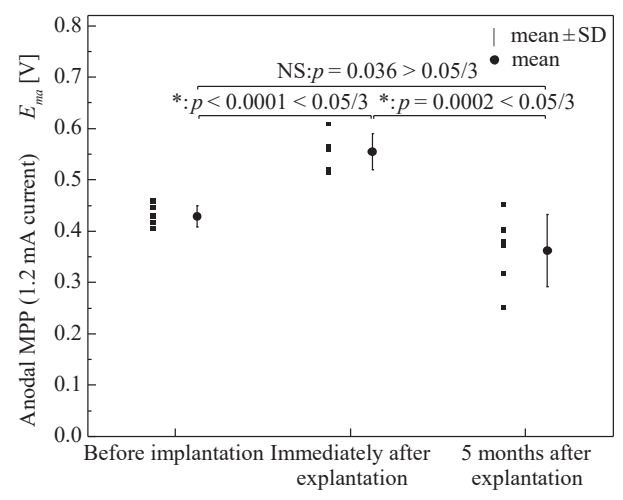

(e)

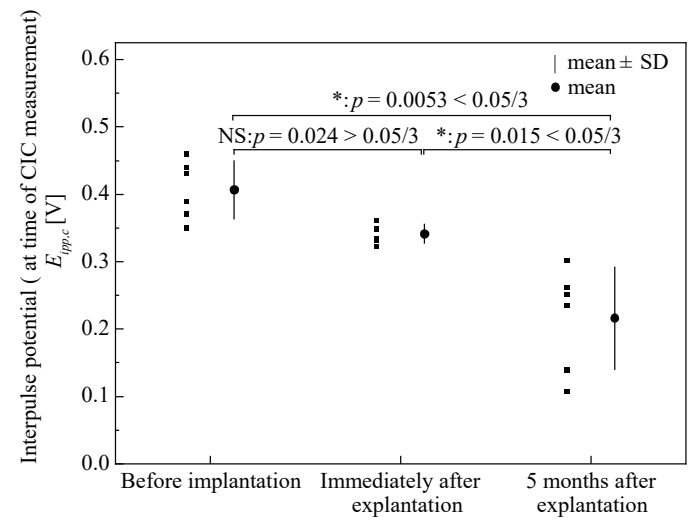

(b)

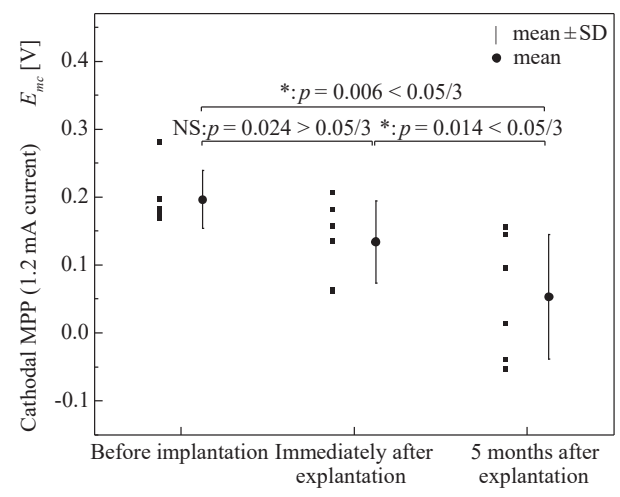

(d)

Fig. 4. In vitro electrochemical evaluation values of electrodes cleaned with $\mathrm{KOH}$ after in vivo testing: before implantation, immediately after explantation, and 5 months after explantation. (a) CIC. (b) Interpulse potential at the time of CIC measurement, $E_{i p p, c}$. (c) Interpulse potential at the time of applying $1.2 \mathrm{~mA}$ current, $E_{i p p, m}$. (d) MPP on the cathodal side at the time of applying $1.2 \mathrm{~mA}$ current, $E_{m c}$. (e) MPP on the anodal side at the time of applying $1.2 \mathrm{~mA}$ current, $E_{m a}\left[^{*}=\right.$ significant $(p \leq 0.05 / 3)$, NS $=$ not significant $(p>0.05 / 3$, paired $t$-test with Bonferroni correction)]. 


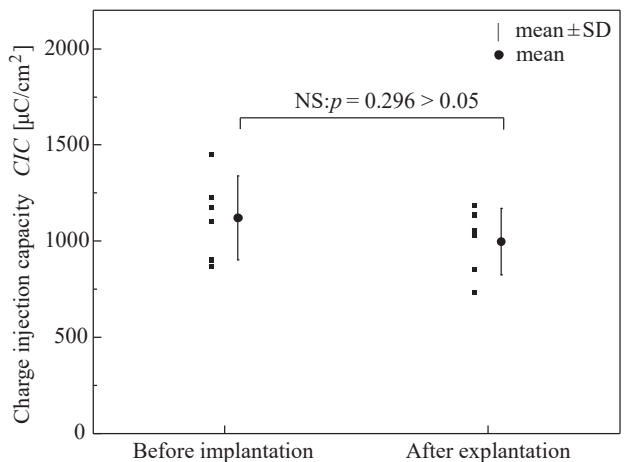

(a)

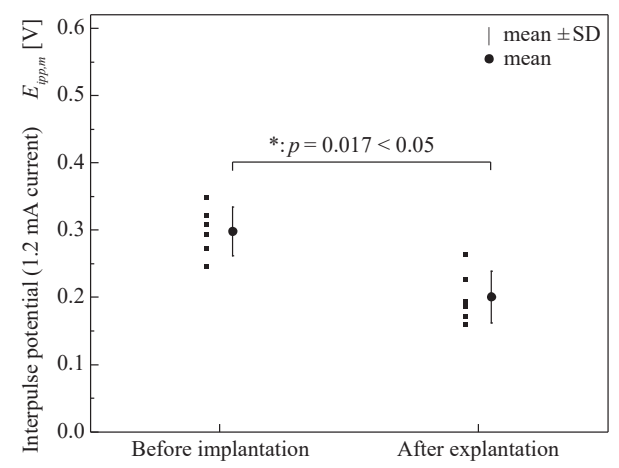

(c)

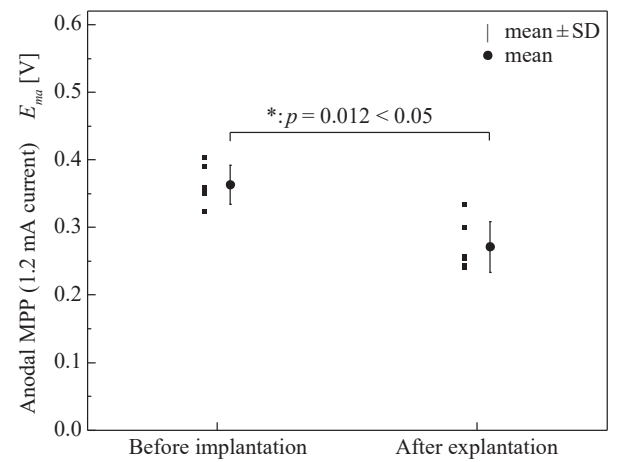

(e)

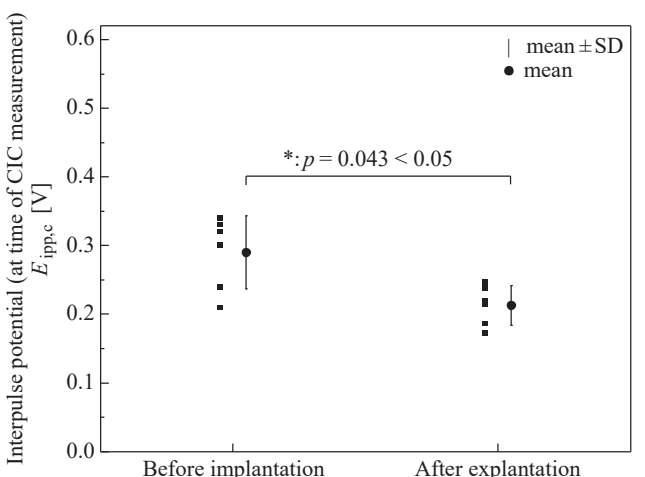

(b)

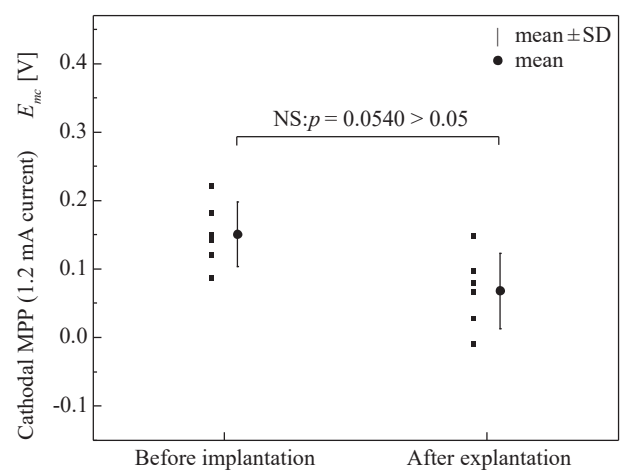

(d)

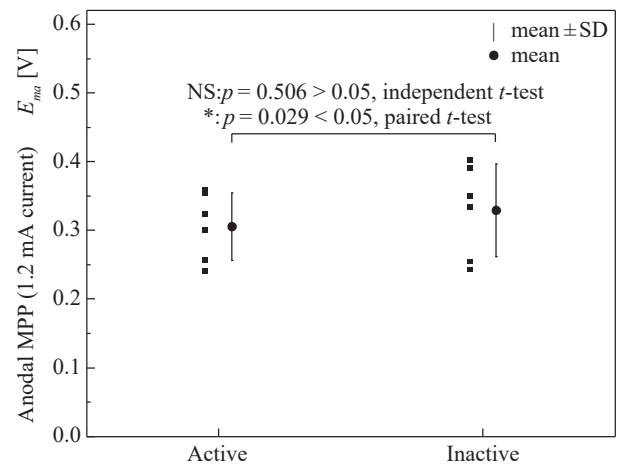

(f)

Fig. 5. In vitro electrochemical evaluation values of electrodes cleaned with Enz. solution after in vivo testing: before implantation and after explantation. (a) CIC. (b) Interpulse potential at the time of CIC measurement, $E_{i p p, c}$. (c) Interpulse potential at the time of applying $1.2 \mathrm{~mA}$ current, $E_{i p p, m}$. (d) MPP on the cathodal side at the time of applying $1.2 \mathrm{~mA}$ current, $E_{m c}$. (e) MPP on the anodal side at the time of applying $1.2 \mathrm{~mA}$ current, $E_{m a}$. (f) $E_{m a}$ for the active electrode and inactive electrode $[*=\operatorname{significant}(p<0.05), \mathrm{NS}=$ not significant $(p>0.05$, paired $t$-test)]. 
of stimulation. Therefore, for $E_{m a}$, the time change using a one-way rANOVA can be applied even if the grouping is active/inactive. Figure 5 shows the in vitro electrical evaluation values of electrodes cleaned with the Enz. solution after in vivo stimulation. The values of CIC and $E_{m c}$ did not change with the adaptation of electrical stimulation in vivo. The values of $E_{i p p}$ and $E_{m a}$ decreased after in vivo use. Figure 6 shows in vitro electrochemical impedance at each frequency after in vivo experimental use. The value of the impedance at each frequency did not change with the adaptation of in vivo stimulation.

The two-way rANOVA was conducted to confirm the changes in the electrochemical characteristics in vivo. There was no primary effect between the adaptation and nonadaptation of stimulation in vivo, except that of impedance. The measurement results of impedance show an interaction between the temporal change and the adaptation/nonadaptation of stimulation current. As the primary effect and interaction with the adaptation/nonadaptation of stimulation were not observed except in the results of the impedance measurement, active and inactive electrodes were grouped together, and the time change was compared using a one-way

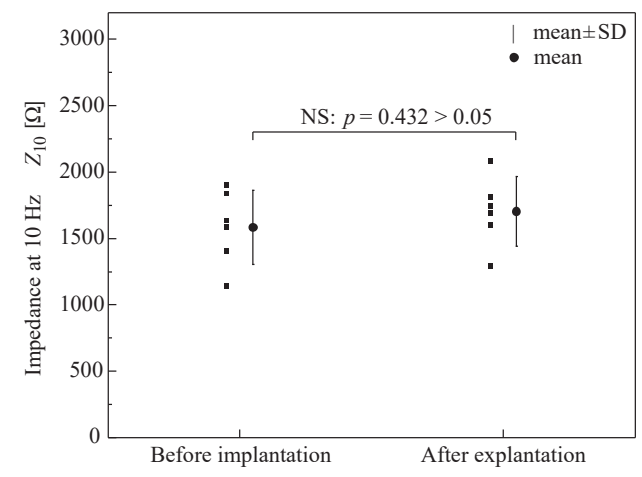

(a)

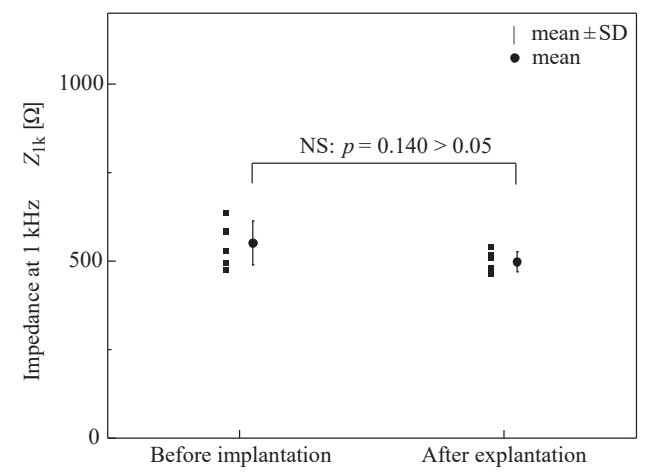

(c)

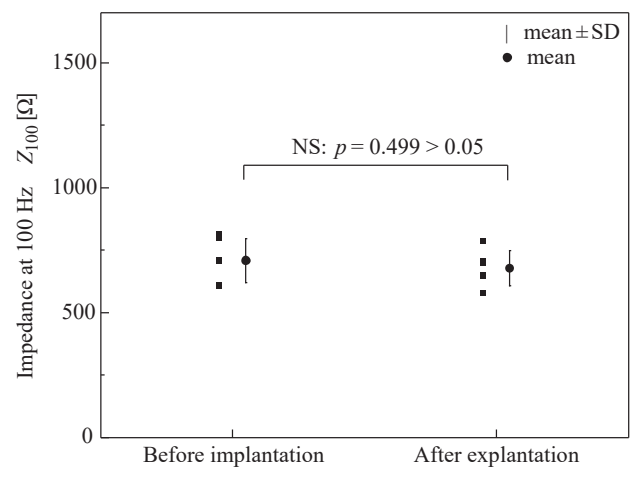

(b)

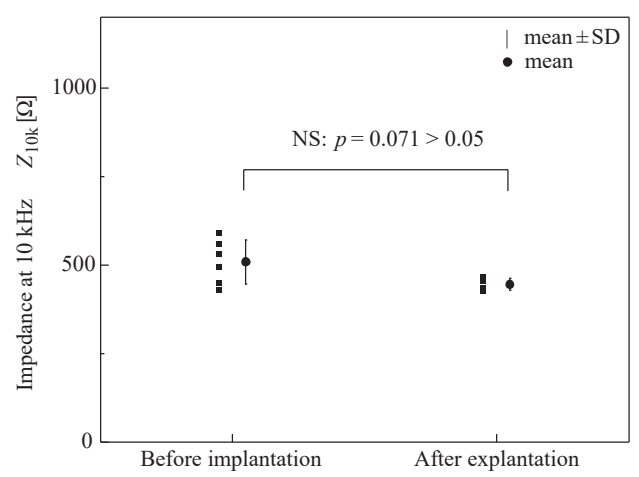

(d)

Fig. 6. In vitro electrochemical impedance of electrodes cleaned with Enz. solution after in vivo testing: before implantation and after explantation. (a) Impedance at $10 \mathrm{~Hz}, \mathrm{Z}_{10}$. (b) Impedance at $100 \mathrm{~Hz}, \mathrm{Z}_{100}$. (c) Impedance at $1 \mathrm{kHz}, \mathrm{Z}_{1 \mathrm{k}}$. (d) Impedance at $10 \mathrm{kHz}, \mathrm{Z}_{10 \mathrm{k}}[\mathrm{NS}=$ not significant $(p>0.05$, paired $t$-test $)]$. 
rANOVA excluding the impedance measurement results (Fig. 7). The in vivo value of CIC was approximately $100 \mu \mathrm{C} / \mathrm{cm}^{2}$, which is $1 / 10$ of the in vitro value [mean $103 \mu \mathrm{C} / \mathrm{cm}^{2}$, standard deviation (SD) $25.6 \mu \mathrm{C} / \mathrm{cm}^{2}$ ]. The $\mathrm{CIC}$ value did not change throughout the in vivo experiment [Fig. 7(a)]. The values of $E_{i p p}$ and $E_{m a}$ decreased rapidly after starting the stimulation and then increased slightly. In contrast, the value of $E_{m c}$ rapidly decreased after the beginning of stimulation and then decreased slightly thereafter. The threshold value of $E_{i p p}$ at the time of CIC measurement regarding whether the non-Faradaic reaction was restricted on the anodal side or the cathodal side was approximately $0.16 \mathrm{~V}$, which is smaller than the in vitro value [Fig. 3(b)]. From the impedance result, interaction was observed between the adaptation/ nonadaptation of stimulation and the time course. Therefore, the temporal change in the active and inactive electrodes was separately analyzed with a one-way rANOVA (Fig. 8). Table 2 shows the results of the correlation analysis of the change in the impedance with time, excluding the data before the stimulation, for the electrode for which the number of missing values was small owing to the stable daily automatic measurement. In the active electrode, the impedance at each frequency decreases rapidly at the beginning of stimulation. Thereafter, the impedance at the low frequency $(10 \mathrm{~Hz})$ decreases slightly, and the impedance at the high frequency $(10$ $\mathrm{kHz}$ ) increases slightly (Fig. 8 and Table 2). A similar tendency was observed in the inactive electrode. However, as the variation of the impedance was large and unstable, no statistically significant difference with respect to the time course was observed.

\section{Discussion}

The in vitro electrochemical properties before and after the in vivo experiments were different: the CIC decreased and $E_{i p p}$ varied in the electrode cleaned with $\mathrm{KOH}$ after explantation (Fig. 4). This result was also presented in several previous reports. ${ }^{(16-18)}$ However, for the electrodes cleaned with Enz. solution after explantation, the in vitro CIC values did not differ before and after the in vivo tests. The differences in the electrochemical properties before and after adaptation of the stimulation current in vivo, as observed in Fig. 4 and the result of two-way ANOVA, are considered to be caused by cleaning with the strong alkaline solution, and were not affected by applying the current. Although the electrochemical properties did not return to their original state even after approximately half a year, the hydroxyl groups on the electrode surface caused by alkali cleaning were replaced by an oxide film over time. When investigating the changes in the electrochemical properties after the in vivo tests, cleaning with acids or bases that affect the electrochemical properties of electrodes should be avoided. The results from the Enz.-cleaned electrode revealed that the electrode performance does not deteriorate even if a stimulation current in the range in which the Faradaic reaction occurs is applied over a long period in vivo (Figs. 5 and 6). When the stimulation current is applied in an oxygen-poor environment, $E_{i p p}$ becomes more negative. ${ }^{(19,20)}$ Because the stimulation current was applied for a long time in a situation where oxygen necessary for the oxidation reaction was depleted, $E_{i p p}$ became more negative and $E_{m c}$ decreased accordingly. When the Faradaic reaction occurs in the chloride solution, $\mathrm{Pt}$ chloride $\left(\mathrm{PtCl}_{4}\right)$ is precipitated on the oxidation reaction side. ${ }^{(19)} \mathrm{As} \mathrm{PtCl}_{4}$ is soluble, no compound film is formed on the electrode surface. 


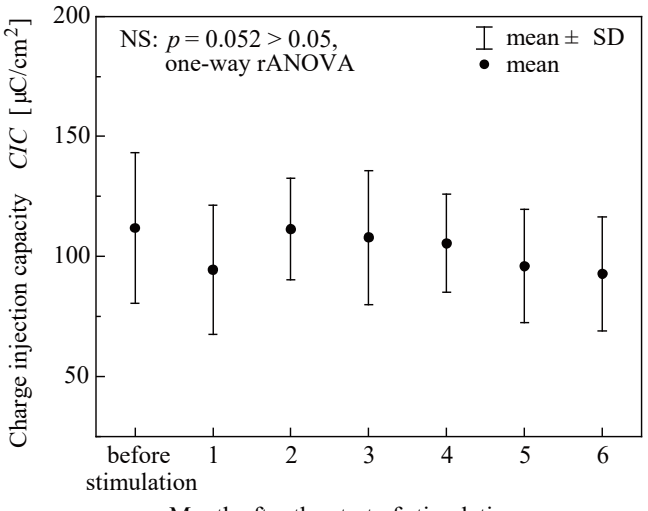

(a)

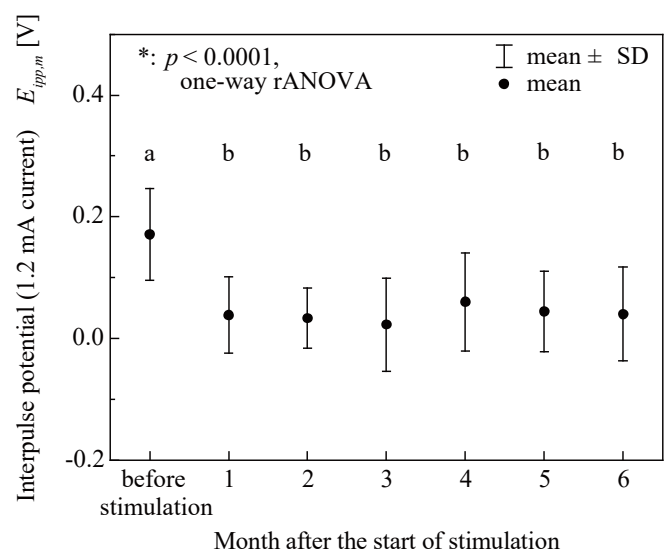

(c)

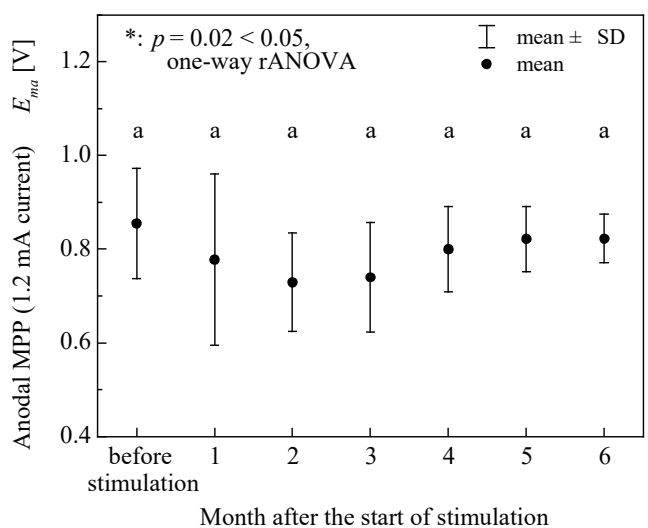

(e)

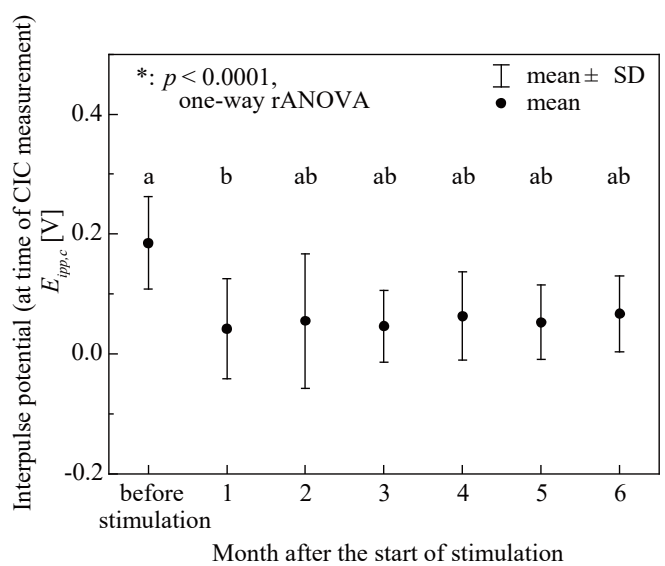

(b)

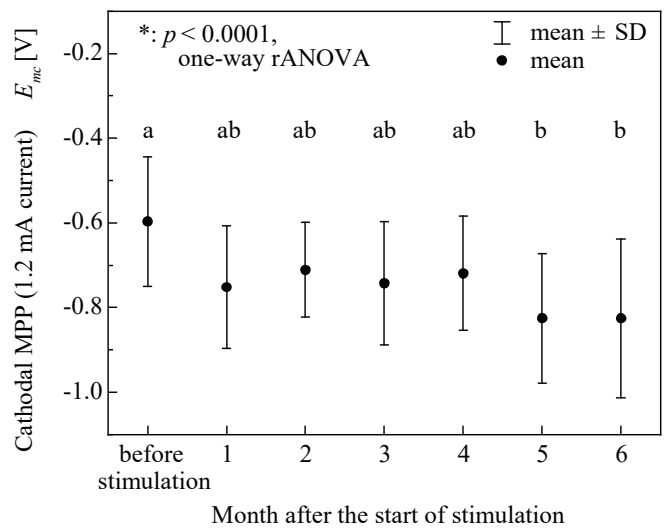

(d)

Fig. 7. Time course of in vivo electrochemical evaluation values. (a) CIC. (b) Interpulse potential at the time of CIC measurement, $E_{i p p, c}$. (c) Interpulse potential at the time of applying $1.2 \mathrm{~mA}$ current, $E_{i p p, m}$. (d) MPP on the cathodal side at the time of applying $1.2 \mathrm{~mA}$ current, $E_{m c}$. (e) MPP on the anodal side at the time of applying 1.2 $\mathrm{mA}$ current, $E_{m a}\left[^{*}=\right.$ significant $(p \leq 0.05), \mathrm{NS}=$ not significant $(p>0.05$, one-way rANOVA)]. The letters a and $\mathrm{b}$ represent significant differences between groups (Tukey HSD test, $p \leq 0.05$ ). The groups indicated by the same letters are not significantly different from each other (Tukey HSD, $p>0.05$ ). 


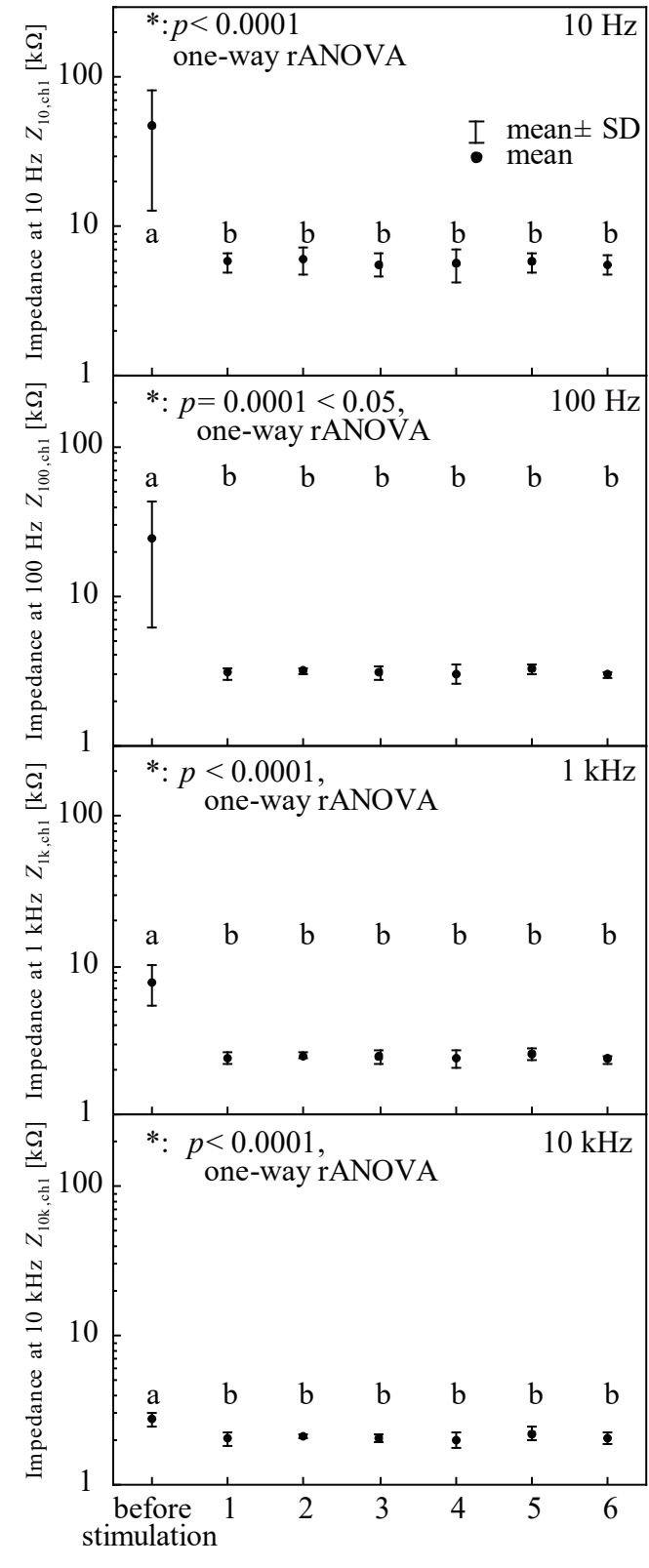

Month after the start of stimulation

(a)

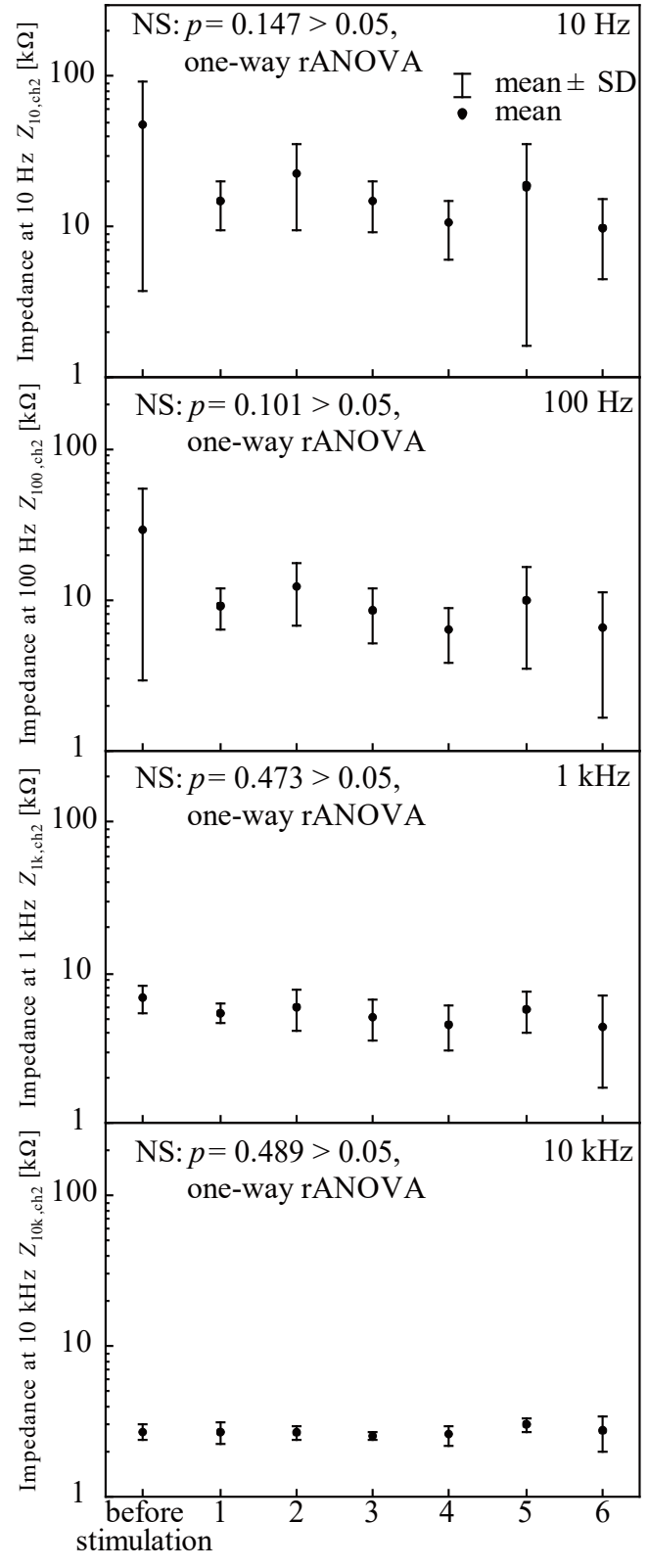

Month after the start of stimulation

(b)

Fig. 8. Time course of in vivo electrochemical impedance $[*=$ significant $(p \leq 0.05)$, NS $=$ not significant $(p>0.05$, one-way rANOVA)]. (a) The result with stimulation current, "active". (b) The result without stimulation current, "inactive". The letters a and b represent significant differences between groups (Tukey HSD test, $p \leq 0.05$ ). The groups indicated by the same letters are not significantly different from each other (Tukey HSD, $p>0.05$ ). 
Table 2

Results of the correlation analysis of the change in in vivo electrochemical impedance with time, excluding the data before the stimulation, for the electrode for which the number of missing values was small owing to stable daily automatic measurement (Pearson correlation coefficient: $r, p$-value of correlation test: $p$ ). "Active" denotes the result with stimulation current, and "inactive" denotes the result without stimulation current. "Total" is the result of analyzing the data obtained by grouping the values of "active" and "inactive" electrodes. "All" is the result of analyzing the data obtained by grouping the values of all the electrodes.

\begin{tabular}{llcccccccc}
\hline \multirow{2}{*}{ Electrode } & \multicolumn{7}{c}{ Frequency } \\
\cline { 3 - 10 } & & \multicolumn{2}{c}{$10 \mathrm{~Hz}$} & \multicolumn{2}{c}{$100 \mathrm{~Hz}$} & \multicolumn{2}{c}{$1 \mathrm{kHz}$} & \multicolumn{2}{c}{$10 \mathrm{kHz}$} \\
\cline { 2 - 10 } No. 1 & Active & 0.399 & $0.07^{\mathrm{NS}}$ & 0.690 & $<0.01^{*}$ & 0.723 & $<0.01^{*}$ & 0.659 & $<0.01^{*}$ \\
& Inactive & -0.304 & $0.18^{\mathrm{NS}}$ & -0.358 & $0.11^{\mathrm{NS}}$ & 0.217 & $<0.01^{*}$ & 0.712 & $<0.01^{*}$ \\
\hline \multirow{2}{*}{ No. 2 } & Active & 0.713 & $<0.01^{*}$ & 0.867 & $<0.01^{*}$ & 0.882 & $<0.01^{*}$ & 0.878 & $<0.01^{*}$ \\
& Inactive & -0.741 & $<0.01^{*}$ & -0.753 & $<0.01^{*}$ & 0.432 & $0.08^{\mathrm{NS}}$ & 0.842 & $<0.01^{*}$ \\
\hline \multirow{2}{*}{ No. 5 } & Active & -0.490 & $0.02^{*}$ & -0.230 & $0.28^{\mathrm{NS}}$ & 0.091 & $0.67^{\mathrm{NS}}$ & 0.457 & $0.03^{*}$ \\
& Inactive & -0.317 & $0.13^{\mathrm{NS}}$ & -0.239 & $0.26^{\mathrm{NS}}$ & 0.173 & $0.42^{\mathrm{NS}}$ & 0.807 & $<0.01^{*}$ \\
\hline \multirow{2}{*}{ No. 6 } & Active & -0.150 & $0.48^{\mathrm{NS}}$ & -0.433 & $0.03^{*}$ & -0.462 & $0.01^{*}$ & -0.513 & $0.01^{*}$ \\
& Inactive & -0.572 & $<0.01^{*}$ & -0.648 & $<0.01^{*}$ & -0.675 & $<0.01^{*}$ & -0.678 & $<0.01^{*}$ \\
\hline \multirow{2}{*}{ Total } & Active & 0.070 & $0.52^{\mathrm{NS}}$ & 0.295 & $<0.01^{*}$ & 0.363 & $<0.01^{*}$ & 0.319 & $<0.01^{*}$ \\
& Inactive & -0.300 & $<0.01^{*}$ & -0.287 & $<0.01^{*}$ & -0.052 & $0.64^{\mathrm{NS}}$ & 0.254 & $0.02^{*}$ \\
\hline All & & -0.178 & $0.02^{*}$ & -0.161 & $0.04^{*}$ & 0.00 & $0.99^{\mathrm{NS}}$ & 0.166 & $0.03^{*}$ \\
\hline
\end{tabular}

*Nos. 3 and 4 had many missing values and were not included in the analysis.

Therefore, it is considered that $E_{m a}$ did not vary despite the change in $E_{i p p}$. The impedance values at each frequency did not vary before and after the adaptation of in vivo electrical stimulation. It is thought that the electrode performance did not deteriorate because the CIC and the electrochemical impedance did not change, although the chemical state of the surface changed with variation of the electrode potential $\left(E_{i p p}\right.$ and $\left.E_{m c}\right)$.

In vivo, a decrease in $E_{i p p}$ occurs regardless of whether the stimulation current is applied. ${ }^{(21)}$ Generally, the restriction on the Faradaic reaction at the Pt electrode is limited on the cathodal side. Therefore, the value of CIC decreases as $E_{i p p}$ becomes more negative. This is because the amount of oxygen required for the reaction is less than that in vitro; the presence of protein, for example, is considered a possible reason, although the actual reason is unknown. ${ }^{(21)}$ After a rapid change postimplantation, the absolute values of $E_{m a}$ and $E_{m c}$ increase despite the stabilization of $E_{i p p}$ (Fig. 7). This is because of the film formation on the Pt surface and the consumption of ions necessary for the reaction around the electrode surface. Such reactivity causes the value of the impedance at low frequencies, although this was unclear from the results because the impedance variation for each electrode is large (Fig. 8 and Table 2). The phenomenon of the electrode impedance rapidly decreasing immediately after applying the stimulation current is also reported in Ref. 22. The changes in the low-frequency components of impedance are due to the changes in charge transfer resistance $\left(R_{c t}\right) .{ }^{(23)}$ However, it is difficult to distinguish $R_{c t}$ from the impedance value of a single frequency because the changes in solution resistance $\left(R_{\text {sol }}\right)$ overlap. Therefore, equivalent circuit analysis via an EIS is beneficial. However, the EIS of a porous electrode 
such as the FLiP electrode is complicated, and the equivalent circuit model is not simple. ${ }^{(24)}$ As the high-frequency component of the impedance bypasses the electric double layer capacity, its value reflects $R_{\text {sol. }}{ }^{(25)}$ The primary reason the high-frequency component of the electrode impedance increases over time is considered to be the encapsulation of the electrode by connective tissues. The increase in solution resistance is not involved in the electrochemical reaction and is related to the potential of the current source. However, the increase is minimal. Therefore, it is not necessary to consider the changes in $R_{\text {sol }}$ when designing the current source. A similar change occurred in the electrode without the stimulation current, although its variation over time was large and slow. On the other hand, as the FLiP electrode was formed by lathe machining from a bulk Pt material and laser irradiation, the surface condition varies considerably between the electrodes. In particular, the characteristics tend to be considerably different before and after maintenance of the laser irradiation apparatus. The oxidation state of the surface considerably influences the electrochemical behavior in vivo. Lathe processing and laser irradiation should be performed under an oxygen-poor atmosphere, if possible, in order to avoid unexpected electrochemical behavior in vivo. If such processing is difficult, it is better to balance the electrochemical characteristics by performing aging treatment with a current for a certain period of time in an electrolytic solution after fabrication. The variation in electrochemical properties may also be due to differences in the crystal structure of the bulk Pt bar, which is used as a raw material. The ratio of in vivo to in vitro CICs of the electrodes without porous processing by femtosecond laser irradiation was reported to be approximately $1 / 4$ to $1 / 5 .^{(26)}$ However, the in vivo CIC reduction of the FLiP electrode compared with that in vitro is approximately $1 / 10$, which is larger than that of the electrode without laser processing. This is due to the porosity of the FLiP electrode surface, although the in vivo performance of the FLiP electrode is higher than that of an electrode without laser processing.

\section{Conclusions}

In this study, we investigated the in vivo electrochemical characteristic of FLiP electrodes for STS-based visual prostheses. The in vivo electrochemical properties of the FliP electrode were stable for over 24 weeks. Cogan et al. argued that appropriate nonclinical and/or clinical data are essential to confirm the safety of the emerging microelectrode with an injected charge density greater than $30 \mu \mathrm{C} / \mathrm{cm}^{2}$. (27) A charge injection of $0.5 \mu \mathrm{C} /$ phase is required for $500 \mu \mathrm{s}$ duration to induce light sensation with our STS retinal prostheses. ${ }^{(6)}$ The maximum output current of the device is $1.2 \mathrm{~mA}(0.6 \mu \mathrm{C} /$ phase at $500 \mu$ s duration). According to Ref. 27, the charge injection in the STS is near the safety threshold. However, the safety threshold of the stimulation current is not uniform as it depends on factors such as the excited cells applied for stimulation, the distance between the cell and stimulation electrode, the configuration of the electrode, the implantation placement of the electrode, and the stimulation parameters. Variations in the electrochemical properties due to the manufacturing method were observed in the FLiP electrode. Therefore, it is better to reduce the variations in electrochemical 
characteristics due to the manufacturing method of FLiP electrodes by using an aging treatment to avoid unexpected side effects of electrical stimulation. Cleaning with a strong acid or a strong base that affects the electrochemical behavior should be avoided. The results of this study include important suggestions on how to confirm electrodes that can be safely used in the process during manufacturing. At the present time, we believe that laser-processed electrodes should be excluded if the electrode potential exceeds the potential window on the anodal side when current equivalent to CIC is applied in PBS. Additionally, CIC in PBS should be over $600 \mu \mathrm{C} / \mathrm{cm}^{2}$ as a guide in this application. However, reliable criteria require comparison between the electrochemical electrode characteristics clarified in this study and the histological evaluation results. In the future, we will investigate and compare the analysis of the electrochemical behavior shown in this paper and the results of the long-term safety tests of STS retinal prosthesis devices.

\section{Acknowledgments}

We thank Professor Takashi Fujikado and Dr. Hiroyuki Kanda of Osaka University for their valuable advice on animal experiments. Moreover, we also thank Masayoshi Nakatani, and Masayuki Shinomiya of Nidek Co., Ltd., and Associate Professor Takashi Tokuda and Dr. Toshihiko Noda of Nara Institute of Science and Technology for their advice and aid in the experiments.

\section{References}

1 A. Brandli, C. D. Luu, R. H. Guymer, and L. N. Ayton: Eye Brain 8 (2016) 15.

2 A. C. Ho, M. S. Humayun, J. D. Dorn, L. da Cruz, G. Dagnelie, J. Handa, P.-O. Barale, J.-A. Sahel, P. E. Stanga, F. Hafezi, A. B. Safran, J. Salzmann, A. Santos, D. Birch, R. Spencer, A. V. Cideciyan, E. de Juan, J. L. Duncan, D. Eliott, A. Fawzi, L. C. O. de Koo, G. C. Brown, J. A. Haller, C. D. Regillo, L. V. Del Priore, A. Arditi, D. R. Geruschat, and R. J. Greenberg (the Argus II Study Group): Ophthalmology 122 (2015) 1547.

3 K. Stingl, K. U. Bartz-Schmidt, D. Besch, A. Braun, A. Bruckmann, F. Gekeler, U. Greppmaier, S. Hipp, G. Hörtdörfer, C. Kernstock, A. Koitschev, A. Kusnyerik, H. Sachs, A. Schatz, K. T. Stingl, T. Peters, B. Wilhelm, and E. Zrenner: Proc. Biol. Sci. 280 (2013) 20130077.

4 H. Kanda, T. Morimoto, T. Fujikado, Y. Tano, Y. Fukuda, and H. Sawai: Invest. Ophthalmol. Vis. Sci. 45 (2004) 560.

5 L. N. Ayton, P. J. Blamey, R. H. Guymer, C. D. Luu, D. A. X. Nayagam, N. C. Sinclair, M. N. Shivdasani, J. Yeoh, M. F. McCombe, R. J. Briggs, N. L. Opie, J. Villalobos, P. N. Dimitrov, M. Varsamidis, M. A. Petoe, C. D. McCarthy, J. G. Walker, N. Barnes, A. N. Burkitt, C. E. Williams, R. K. Shepherd, and P. J. Allen (the Bionic Vision Australia Research Consortium): PLoS One 9 (2014) e115239.

6 T. Fujikado, M. Kamei, H. Sakaguchi, H. Kanda, T. Endo, M. Hirota, T. Morimoto, K. Nishida, H. Kishima, Y. Terasawa, K. Oosawa, M. Ozawa, and K. Nishida: Invest. Ophthalmol. Vis. Sci. 57 (2016) 6147.

7 M. Mahadevappa, J. D. Weiland, D. Yanai, I. Fine, R. J. Greenberg, and M. S. Humayun: IEEE Trans. Neural Syst. Rehabil. Eng. 13 (2005) 201.

8 E. Zrenner, K. U. Bartz-Schmidt, H. Benav, D. Besch, A. Bruckmann, V.-P. Gabel, F. Gekeler, U. Greppmaier, A. Harscher, S. Kibbel, J. Koch, A. Kusnyerik, T. Peters, K. Stingl, H. Sachs, A. Stett, P. Szurman, B. Wilhelm, and R. Wilke: Proc. Biol. Sci. 278 (2011) 1489.

9 Y. Terasawa, H. Tashiro, Y. Nakano, T. Tokuda, and J. Ohta: Adv. Biomed. Eng. 5 (2016) 137.

10 Y. Terasawa, H. Tashiro, K. Osawa, M. Ozawa, T. Noda, J. Ohta, and T. Fujikado: Invest. Ophthalmol. Vis. Sci. 53 (2012) 5538.

11 T. Fujikado: Artificial Vision - A Clinical Guide, ed. V. P. Gabel (Springer International, Cham, 2017) Chap. 11. 
12 S. F. Cogan: Annu. Rev. Biomed. Eng. 10 (2008) 275.

13 The Association for Research in Vision and Ophthalmology: http://www.arvo.org/About_ARVO/Policies/ Statement_for_the_Use_of_Animals_in_Ophthalmic_and_Visual_Research/(accessed July 2017).

14 Y. Terasawa, H. Tashiro, Y. Nakano, and J. Ohta: Sens. Mater. 2 (2018) 235.

15 H. Tashiro, Y. Terasawa, M. Kuwabara, K. Osawa, T. Tokuda, J. Ohta, and T. Fujikado: Adv. Biomed. Eng. 6 (2017) 8.

16 Y. Terasawa, H. Tashiro, Y. Nakano, K. Osawa, and M. Ozawa: Invest. Ophthalmol. Vis. Sci. 54 (2013) 1054.

17 Y. Terasawa, H. Tashiro, Y. Nakano, K. Osawa, M. Ozawa, T. Noda, T. Tokuda, J. Ohta, and T. Fujikado: Invest. Ophthalmol. Vis. Sci. 55 (2014) 1803.

18 Y. Terasawa, H. Tashiro, Y. Nakano, K. Osawa, M. Ozawa, T. Noda, T. Tokuda, J. Ohta, and T. Fujikado: Invest. Ophthalmol. Vis. Sci. 56 (2015) 758.

19 D. R. Merrill, M. Bikson, and J. G. R. Jefferys: J. Neurosci. Methods 141 (2005) 171.

20 R. T. Leung, M. N. Scivdasani, D. A. X. Nayagam, and R. Shepherd: IEEE Trans. Biomed. Eng. 62 (2015) 849.

21 S. F. Cogan: Proc. IEEE Eng. Med. Biol. Soc. 2006 (2006) 882.

22 K. J. Otto, M. D. Johnson, and D. R. Kipke: IEEE Trans. Biomed. Eng. 53 (2003) 333.

23 A. Mercanzini, P. Colin, J. C. Bensadoun, A. Bertsch, and P. Renaud: IEEE Trans. Biomed. Eng. 56 (2009) 1909.

24 M. Kuwabara, H. Tashiro, Y. Terasawa, Y. Nakano, T. Noda, T. Tokuda, and J. Ohta: Proc. 39th Annu. Int. Conf. IEEE Engineering in Medicine and Biology Society (IEEE, 2017) FrDT7-09.1.

25 C. Henle, M. Raab, J. G. Cordeiro, S. Doostkam, A. Schulze-Bonhage, T. Stieglitz, and J. Rickert: Biomed. Microdevices 13 (2011) 59.

26 H. Tashiro, Y. Terasawa, K. Osawa, M. Ozawa, T. Noda, J. Ohta, and T. Fujikado: Invest. Ophthalmol. Vis. Sci. 53 (2012) 5518.

27 S. F. Cogan, K. A. Ludwig, C. G. Welle, and P. Takmakov: J. Neural Eng. 13 (2016) 021001. 